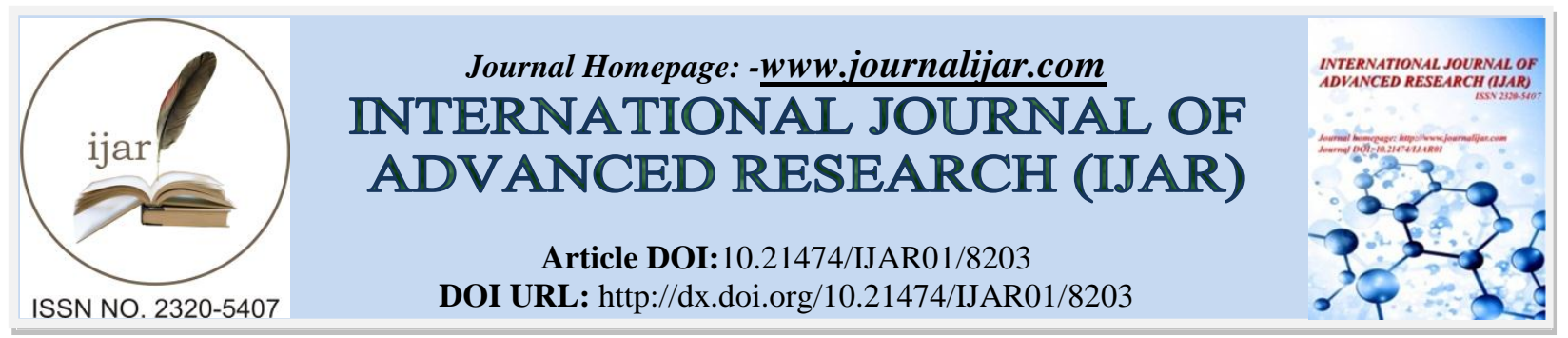

RESEARCH ARTICLE

\title{
DESIGN OF WIRELESS SENSOR NETWORK FOR AIRCRAFT HEALTH MONITORING.
}

\author{
Zhang Chuanchao. \\ School of Information Engineering,Wuhan University of Technology, Wuhan 430070,P.R.China,Aviation \\ Industry Corporation of China,Ltd., Beijing 100028, PR.China.
}

\section{Manuscript Info}

Manuscript History

Received: 12 October 2018

Final Accepted: 14 November 2018

Published: December 2018

\section{Abstract}

In view of the problems such as wiring difficulties, a large number of sensors and the wiring not only to increasing the mass but also occupying the space of aircraft for health monitoring using wired network transmission, combining with the characteristic of function demand and regional management, heterogeneous data in aircraft health monitoring, taking advantage of wireless sensor, a system solution based on wireless sensor network for aircraft health monitoring is put forward, and the overall architecture, node hardware and software protocol, network topology structure are designed in this paper.

Copy Right, IJAR, 2019,. All rights reserved.

\section{Introduction:-}

A large number of sensors were installed in the aircraft airborne systems, one part of them were used for aircraft, engines and mechanical and electrical system control. Another part was used for health status and fault monitoring for aircraft subsystems and its key components. At present, most of these sensors are in the wired connection way, which has brought serious problems in the aircraft system design and maintaining. The mainly effects are as follows:

Aircraft system had a large number of transmission lines, which had not only limited the interior space of the aircraft, but also increased wiring engineering, which seriously affects the design, installation and debug of the aircraft.

A large number of transmission lines have brought greater difficulties to the maintenance of the aircraft system. If a problem occurs, it takes a long time to find the fault source. A lot of wiring have not only increased the weight, but also occupied the space of the aircraft, and making the aircraft's limited space become more narrow. At the same time, reducing the transmission lines can also reduce the weight, expand the internal space.

The number of sensors has bring the increasing quality of the aircraft, and even affected the performance of aircraft parts, thereby affected the flight safety. Although non-contact sensors have been used[1], but the selection and optimization of sensor layout are very difficult problems.

The existing airborne systems are usually faced with limited space, many types of the monitoring parameters, large amount of data, strong electromagnetic interference and real-time transmission information and harsh working environment, etc. This sensor technology proposed requirements are as follows: small size, light weight, easy installation and maintenance, convenient system and preprocessing unit network, not easily affected by electromagnetic interference, adapting to the harsh working conditions and environment, etc. There is an urgent need for a wireless embedded and distributed data mining processing method to solve data collection problems of prognostics and health management (PHM) system, and to further reduce costs and improve system reliability [2].

Corresponding Author:-Zhang Chuanchao.

Address:-School of Information Engineering,Wuhan University of Technology, Wuhan 
Compared with the traditional wired sensor networks, wireless sensor networks have obvious advantages[3]. Taken the Seahawk SH60 as an example, aiming at the control system using the wireless sensor network technology, reducing $50 \%$ cable of the control system can reduce about $121 \mathrm{~kg}$ weight, while not only can increase weapons load, also can reduce fuel consumption and increase the mileage. It can been seen, compared with the traditional wired sensor networks, wireless sensor network has advantages, such as low cost, no wiring, convenient installation, and is very suitable for difficult wiring occasions ,such as aircraft structures, engine, electrical and mechanical systems, can solve the traditional wired sensor wiring difficulties, poor flexibility, high cost, accident difficult to isolate and other practical problems, provide reliable data for aircraft health monitoring.

Wireless sensor networks is an emerging paradigm of computing and networking where a node may be self powered, and have sensing, computing, and communication capabilities. They have been proposed for use in a wide variety of applications for airplane monitoring. Literature [4] describes a wireless sensor network for monitoring of the health of aircraft engines and describes the architecture of the wireless sensor network along with how it fits into the general area of wireless sensor networks.

In traditional airplane monitoring system (AMS), data sensed from strain, vibration, ultrasound of structures or temperature, and humidity in cabin environment are transmitted to central data repository via wires. However, drawbacks still exist in wired AMS such as expensive installation and maintenance, and complicated wired connections. In recent years, accumulating interest has been drawn to performing AMS via airborne wireless sensor network (AWSN) system with the advantages of exibility, low cost, and easy deployment. Literature [5] present an overview of AMS and AWSN and demonstrate the requirements of AWSN for AMS particularly. Furthermore, existing wireless hardware prototypes and network communication schemes of AWSN are investigated according to these requirements. The paper will improve the understanding of how the AWSN design under AMS acquires sensor data accurately and carries out network communication, providing insights into prognostics and health management (PHM) for AMS in future.

Network protocol is one of the key points of current airborne detection system researches. For the communication requirements of real-time, reliability, and energy consumption of clustering wireless sensor networks for aircraft health monitoring, literature [6] proposes a modified TDMA protocol and relevant cluster members time synchronization mechanism. This modified TDMA protocol can adaptively allocate time slots based on sensor nodes data traffic, so that it can improve using efficiency of the channel, reduce the loss rate of the packets, time delay, and energy consumption. Along with the time slots, cluster head broadcasts its time stamps to sensor nodes, which adopt linear regression analysis method to estimate the relative drift and relative deviation of the clock. Then the sensor node could adjust its clock according to the head.

Information fusion is one of the key points of current airborne detection system researches. In literature [7], the use of an ad hoc wireless sensor network for implementing a structural health monitoring system is discussed. The network is consisted of sensors deployed throughout the aircraft. These sensors being in the form of a microelectronic chip and consisted of sensing, data processing and communicating components could be easily embedded in any mechanical aircraft component. The established sensor network, due to its ad hoc nature is easily scalable, allowing adding or removing any number of sensors. The position of the sensor nodes need not necessarily to be engineered or predetermined, giving this way the ability to be deployed in inaccessible points. Information collected from various sensors of different modalities throughout the aircraft is then fused in order to provide a more comprehensive image of the aircraft structural health. Sensor level fusion along with decision quality information is used, in order to enhance detection performance.

Energy supply is one of the key points in the researches of airborne detection system. Literature [8] reports a selfpowered WSN system based on piezoelectric energy harvesting and used for aircraft health monitoring. The system consists of three main subsystems, energy harvesting unit, MCU \& wireless transceiver unit, and sensors unit. The prototype has dimension of $138 \mathrm{~mm}(\mathrm{~L}) \times 41 \mathrm{~mm}(\mathrm{~W}) \times 23 \mathrm{~mm}(\mathrm{H})$ with a weight of only 40grams, making it easily integrated and implemented in an aircraft environment. Simulation results showed that with an excitation of $40 \mathrm{~Hz}$ frequency and $0.5 \mathrm{~g}$ acceleration on the harvester, the system takes about $3.4 \mathrm{secs}$ to power the system with a selected voltage and another $23 \mathrm{secs}$ to perform the initialization operations of the system. After the initialization, data are 
measured and transmitted wirelessly with a total energy consumption of $0.65 \mathrm{~mJ}$ in one cycle every one second. In addition, a new power management strategy for the applications using capacitors to store harvested energy was proposed. The mechanism relies on the analysis of how much energy has been stored on capacitor to determine the executions of the operations in the system. A novel energy saving interface (ESI) is designed to minimize the power consumption during the voltage measurement and an intelligent algorithm was developed to implement the strategy. Initial experiments show that the adapted Z-Stack and the proposed algorithm can be implemented on the system designed in this paper. Literature [9] presents a wireless sensor node (WSN) powered by a strain energy harvester (SEH) through an adaptive power management module (PMM) for aircraft structural health monitoring (SHM). The energy distribution in the system, the efficiencies of the whole systems, and the WSN powering capability of the SEH under different strain loads were studied to understand the developed system performance for practical applications of an autonomous WSN. Experimental results show that the SEH is able to produce up to $3.34 \mathrm{~mW}$ under strain loading of $600 \mu \varepsilon$ at $10 \mathrm{~Hz}$. The WSN can be powered up through the adaptive PMM at efficiency from $70 \%$ to $80 \%$ under different test conditions.

Time synchronization is one of the key points of current airborne detection system researches. Energy harvesting, combined with wireless sensors, could greatly improve our ability to monitor and maintain critical structures. Literature [10] reports on the development of an integrated structural health monitoring and reporting (SHMR) system for use on Navy aircraft. Its goal was to develop and test a versatile, fully programmable SHMR system, designed to synchronize and record data from a range of wireless and hardwired sensor networks. Wireless sensors included strain gauges, accelerometers, and thermocouples. Hard-wired sensors included gyroscopes, accelerometers, and magnetometers. Data from an embedded Global Positioning System (GPS) provided position, velocity, and precise timing information.

Wireless sensor network technology has been studied in aircraft subsystems such as aeroengine [4], control system[11], structure system[12,13,14,15] and pilot system[16]. Among them, structural health monitoring is the most studied. Use of fly-by-wire technology for aircraft flight controls have resulted in an improved performance and reliability along with achieving reduction in control system weight. Implementation of full authority digital engine control has also resulted in more intelligent, reliable, light-weight aircraft engine control systems. Greater reduction in weight can be achieved by replacing the wire harness with a wireless communication network. The first step towards fly-by-wireless control systems is likely to be the introduction of wireless sensor networks (WSNs). WSNs are already found a variety of applications for both safety-critical and no safety-critical distributed systems. Some of the many potential benefits of using WSN for aircraft systems include weight reduction, ease of maintenance and an increased monitoring capability. Literature [11] discusses the application of WSN for several aircraft systems such as distributed aircraft engine control, aircraft flight control, aircraft engine and structural health monitoring systems. The current trend towards the use of novel materials and design concepts for aircraft structures may demand structural health monitoring (SHM) systems in the future. Wireless sensor networks could fulfill the required monitoring tasks concerning fatigue, damage or stress of structural parts. Autonomous sensor nodes (ASNs) are key elements for a WSN in order to allow a self-sufficient and maintenance free operation, without any complex wiring for power supply or communication purposes [14]. Literature [12] discusses an architecture and design of a sensor network to be used for structural health monitoring of an aircraft. Also presented are several prototypes of critical parts of the sensor web. The proposed sensor network will utilize sensor nodes situated throughout the structure. These nodes and one or more workstations will support agents that communicate and collaborate to monitor the health of the structure. Agents can be any internal or external autonomous entity that has direct access to affect a given system. For the purposes of this document, an agent will be defined as an autonomous software resource that has the ability to make decisions for itself based on given tasks and abilities while also collaborating with others to find a feasible answer to a given problem regarding the structural health monitoring system. Once the agents have received relevant data from nodes, they will utilize applications that perform data fusion techniques to classify events and further improve the functionality of the system for more accurate future classifications. Agents will also pass alerts up a self-configuring hierarchy of monitor agents and make them available for review by personnel. This thesis makes use of previous results from applying the Gaia methodology for analysis and design of the multi-agent systems. Structural health monitoring is a critical part of the maintenance procedures of an aircraft. Most of the problems that arise from structural elements are detected by trained personnel using visual inspection. Moreover, the places at which these structural elements are located are frequently very difficult to access. This makes inspections time consuming and 
potentially very costly. Literature [15] are presenting the design principles that must be taken into account in order to successfully deploy a wireless sensor network in an aircraft. It discusses the relations between sampling duration, power consumption, energy harvesting and storage, wireless communication and network architecture in order to successfully deploy the system. In order to consider the trade-offs between different design issues it have designed a sensor node equipped with an accelerometer, a temperature and humidity sensor and a strain gauge sensor. The sensor node has an energy harvester from a thermo-electrical generator and a battery storage element. Wireless communications are based on Zigbee protocol and the sensor node is controlled from a low power microcontroller. In literature [13], critical event-triggered transmission scheme is illustrated and applies to prolong the battery lifetime and balance the power-performance tradeoff. The experimental results show that the wireless sensor node using this technology is a powerful solution for aircraft SHM. Wireless sensor networks can be used to improve both safety critical and unsafety critical aircraft systems. Using wireless sensor networks can help to increase the number of sensors as well the system redundancy and also helps to reduce the aircraft system weight and complexity, improving the fuel efficiency and maintenance costs. Supporting standard protocols in all wireless sensor nodes simplifies the application development, configuration and maintenance. The wireless sensor network devices can also be used to monitor the physiological pilot's parameters. Literature [16] presents a complete and innovator solution, mainly based on standard protocols, to monitor light aircraft and gliders pilot's physiologic parameters. The proposed system does not interfere with pilot's agility, is simple to install, configure and operate. To evaluate the system, a real testbed was deployed.

However, the application of wireless sensor technology in aircraft health monitoring is a complex system engineering, involving not only all subsystems, but also a series of technical problems [17]. Current research only focuses on one of the subsystems, which does not meet the implementation requirements. Real aircraft health monitoring is based on the aircraft as the whole research object, which requires a complete technical framework. This is conducive to the design according to the partition principle of aircraft health management, but also conducive to time synchronization. Based on the partition principle of aircraft health management [18], this paper designs a complete aircraft health monitoring system architecture.

\section{Requirements for aircraft health monitoring:-}

In order to meet the requirements of the use and maintain, the aircraft health monitoring needs to achieve the following functions:

1. fault detection, fault isolation and performance monitoring.

2. fault prediction of critical systems and components.

3. life prediction of the residual service.

4. tracking for the service life of parts.

5. trend tracking for performance degradation.

6. selective reporting of the failure.

7. assistant decision-making and resource management.

Typically, an aircraft has main parts of the engine system, mechanical and electrical system, avionics system and structure system. The health monitoring needs of each part[19] are as follows.

\section{Engine system:-}

The requirements for engine health monitoring are as follows:-

Engine vibration management. Through the analysis of the vibration signal, the fusion information of gas, oil and other sources, the rotor system vibration faults can be isolated at least to high pressure rotor and low pressure rotor, and drive system.

Oil system management. By monitoring the pressure and temperature of oil and the number of particles in the oil, the health state of the lubricating oil system and lubricating parts in the transmission system are monitored. 
Control and fuel system management. Though the measurement data, the conclusion of the monitoring and diagnosis data of the control system, the test results of the fuel system parameters, the fault diagnosis of the control system and the fuel oil system can be carried out.

Life management. The engine management unit of life and the life of critical components are managed. To monitor key parts (attached) use life and remaining life monitoring, computing the low cycle fatigue (LCF) of the cold end rotating parts and creep/fatigue life of turbine blade and automatic recording cycle number of life, calendar life, flight hour life, the starting number life, and so on.

\section{Mechanical and electrical system:-}

Mechanical and electrical system is consisted of hydraulic system, fuel system, power supply system, environmental control and thermal management system, auxiliary engine system, landing gear system, fire protection system, and external lighting system, cabin cover system, weapon bay door drive system and life support systems and other components in general.

Health monitoring requirements for hydraulic systems. Based on the monitoring and analysis for hydraulic pump outlet pressure, flow rate, temperature and vibration, speed and other parameters, implementation hydraulic pump condition monitoring in different loads and different working conditions. Through hydraulic pressure of import and export, traffic monitoring for actuating cylinder, implementation state monitoring for hydraulic actuator. Through oil quality, pressure, vibration, temperature monitoring of key nodes in hydraulic system, implementation monitoring and prediction of common faults for the hydraulic leakage, wear, pressure pulsation. Monitoring logic switching between redundancy system and the normal system in single hydraulic system, ensuring that the redundant hydraulic system can work normally. Monitoring the state of control valve and the logical relation when hydraulic systems linkage, ensuring that the linkage of hydraulic system according to the predetermined logical relationship.

The health monitoring requirements for fuel system. Realize the monitoring for the working state of the various components. To solute tank leakage and blockage, normal, leakage and other problems of gas and oil transporting pipeline in system level. Tank fault diagnosis is very complex, relating to the state of pump, oil level sensor, gas valves, the hint pumps, transmission oil, so gas pipeline failure is also very complex, there is no way to separate judgment, it must be analyzed and judged in system level. Monitoring fuel system status, evaluation function and fault alarm.

The health monitoring requirements for power supply system. Evaluation and prediction of power supply capacity for power supply system. To monitor the working condition of the power supply system, and evaluate and predict the capacity of the power supply system. Tracking and monitoring life, according to the working hours of the actual parts, the remaining life of key components are evaluated and recorded.

The health monitoring requirements for aircraft environmental and control system. The main purpose of fault diagnosis for aircraft environmental and control system (ECS) is to improve the reliability and safety of the aircraft, through real-time monitoring and fault diagnosis to ensure the aircraft cabin and equipment with good environmental parameters in the various flight conditions, to meet the needs of normal life and work of the personnel and equipment. Though flow, temperature, pressure signal and turbine, control valves and other key product state parameters in environmental control and thermal management system, and collection, storage, transmission and management of process parameters, environmental and control system and a finished work state are achieving monitoring.

The health monitoring requirements for aircraft accessory drive system. The fault diagnosis and health management of the accessory drive system need to be realized: (a) Vibration monitoring and warning. (b) Oil system condition monitoring. (c) Fault diagnosis and health assessment. (d) Through the flight state identification and load analysis, the life analysis of key components is realized, which can provide support for maintenance decision.

\section{Avionics system:-}

The health monitoring requirements of avionics system are as follows:-

1. Monitoring the work information (parameters and status) of each subsystem by BIT. 
2. By the embedded micro sensor in avionics equipment, implementation monitoring for environmental stress (temperature, vibration, temperature).

3. The enhancement of fault diagnosis, confirmation, isolation and report. Accepting fault report, status information from the system of all subsystems / components, to take feature extraction, analysis and multi-source information fusion, to confirm fault diagnosis, fault filtering false alarm and evaluation of intermittent faults and accurate fault isolation (located to subsystems) in regional level, and to confirm the fault according to severity classification, to send selective report to the aircraft platform manager in a timely manner.

4. According to the actual situation of the equipment, through the different forecasting methods, it can achieve fault prediction for avionics equipment. For the common faults of the system, it can realize the fault trend analysis, and carry on the appraisal to the current capability system, give the performance evaluation of the degradation.

5. Self reconstruction. When the system component fails, the system is reconstructed. First, due to the change of system functional requirements, the system component is relocated. second, in any part of the system is determined to be the failure, the rest will be redistributed to restore the system function.

\section{Structural system:-}

The structure of fault diagnosis and health management needs to be realized:-

1. Structural overload monitoring (overweight or speeding, heavy landing, structural stress overload, etc.). By monitoring flight parameters, load stress levels, to determine the structural overload situation, to assess the health status of the structure, and the no-scheduled maintenance recommendations of the aircraft is put forward.

2. Evaluation and prediction of structure fatigue life. Based on information and load information, to take preprocessing and feature extraction of information, to determine the course load and stress/strain history of the key parts or structure, to combine with the cumulative damage model for structural damage identification and location, and to assess the cumulative lifetime consumption, and to predict the residual fatigue life.

3. Analysis corrosion of key parts. Though collecting the corrosion information, combining the empirical data, to evaluate and predict the corrosion degree of key parts.

4. Structural health management. To provide fatigue information, structural overload information for the fuselage and key parts, corrosion information of key parts, and to associate with these informations, to manage the life of aircraft structure and key parts. Using the data recorded in the system and historical data, we should be able to carry out the analysis of the structure health status (damage trend) of the single aircraft/aircraft group, realize the life monitoring of the single aircraft and make the structure maintenance plan of aircraft group.

\section{System design:-}

Characteristics of aircraft health monitoring:-

Aircraft health monitoring has the following characteristics:-

Hierarchy. The general composition of aircraft is engine system, mechanical and electrical system, avionics system and structure subsystem. The internal member system of the subsystem has more types and complex relationships, and all levels of the subsystems need to be monitored.

Heterogeneous. According to the requirements analysis of aircraft monitoring, these physical parameters such as pressure, flow and vibration, oil temperature, speed, force, strain and time, must be monitored. The sensor configuration can be divided into temperature sensor, pressure sensor, speed sensor, vibration sensor, displacement sensor and other new sensor.

Partition management. The state health of components determines the health status of the subsystem, and the health status of the subsystem determines the health status of the aircraft platform system. Therefore, from the physical structure, aircraft health management system has used hierarchical structure and distributed mechanism [18], airborne system is divided for three grade management, which are member level, regional level and the aircraft platform level. According to different functional subsystems (for example engine system, structure, mechanical and electrical management system and other systems), special regional manager is designed, and high-level data fusing is achieved in the aircraft manager. From the module and unites to obtain the sensor information, BIT information, through reasoning and analysis of various levels of PHM, the aircraft system health status is gained, and the faults in the system are processed timely.

\section{Overall framework:-}


The wireless sensor network for aircraft health monitoring mainly consists of three parts: sensor node group, communication network and information center. The overall structure of the system is shown in Figure 1. ZigBee technology based on IEEE802.15.4 protocol can be used, which is composed of a number of sensor nodes and a coordinator node to form a basic monitoring area, which is at the bottom of the system. The entire network can be selected according to the needs of the appropriate topology, such as star network, net network and tree network, etc. The terminal node can be configured with single or multiple different precision temperature, pressure, stress and other sensors used for aircraft health monitoring information collection. Terminal nodes are deployed in the monitoring area, each terminal node in a self-organizing way constitute wireless sensor networks, and cooperatively sense and collect network coverage area of aircraft health information. After the primary process of the detected information, monitoring information is relayed to the coordinator node in the single hop or multi hop way. Subsequently, through a dedicated satellite, Internet or public mobile communication network coordinate node will send these information to aircraft monitoring center. Finally, on the basis of monitoring data of aircraft health monitoring, the information center will take integrated management and configuration the wireless sensor network. The relationship between aircraft health monitoring system and wireless sensor unit is shown in Figure 2.

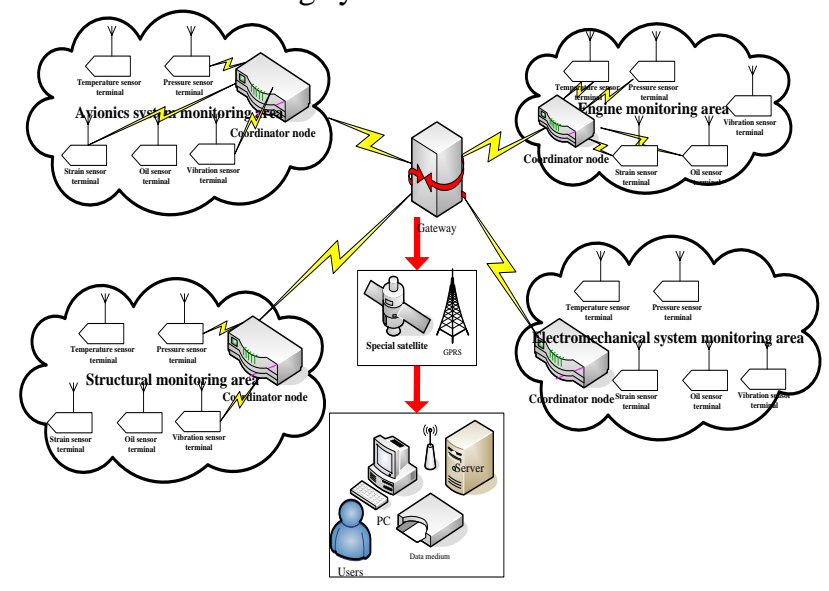

Figure 1:-the overall structure of the wireless sensor network system for aircraft health monitoring

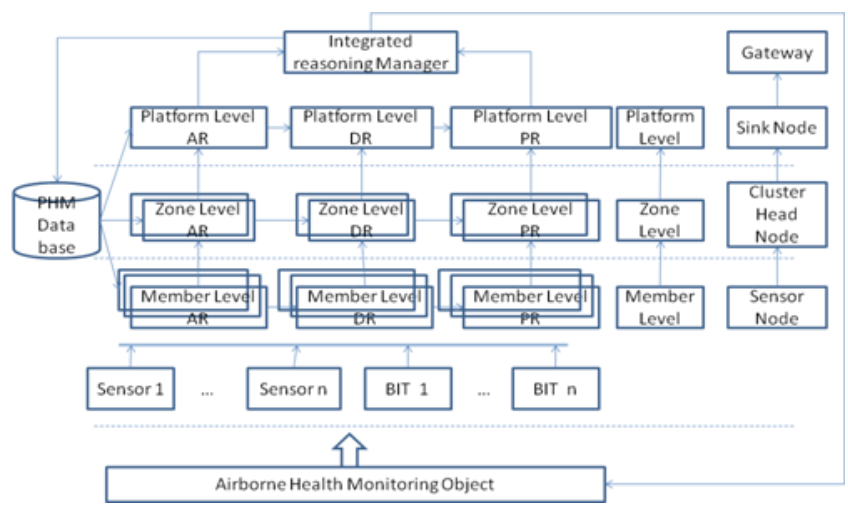

Figure 2:-Relationship between aircraft health monitoring system and wireless sensor unit

\section{Node hardware design:-}

The sensors which are distributed in the monitoring area include many kinds of sensors, such as temperature sensors, pressure sensors and pressure sensors, which are responsible for the real-time collection of different type data. The terminal node directly measured aircraft health monitoring data, after preliminary treatment transmitted the collected data to the coordinator node. It is the sources of information of the real-time monitoring system. A simple sensor terminal node is composed of sensor module, processor module, memory module, RF module and power module. 
In fact, the hardware design of all kinds of sensor terminal nodes is similar, but the specific sensor modules are different. The sensor module is responsible for collecting the aircraft's health data. The microprocessor module is responsible for the control of the processing operation, equipment control, power management and task management for the whole node. The RF module is responsible for wireless communication with other nodes, controlling the exchange of information and receiving and sending the collected data. The memory module is responsible for data storage of the identification information of the sensor itself and the collected aircraft health information. The power supply module ensures the energy supply of the whole terminal node, and satisfies the continuous working for a long time.

The coordinator node is responsible for sending and receiving the data from the data collected by the near sensor nodes, and carrying out the corresponding processing. After receiving the data, the data will be analyzed, fused and preprocessed to ensure the real-time transmission of the emergency signal, so it has strong processing, storage and communication capabilities. The coordinator node can be an enhanced terminal node with sufficient energy supply and more memory resources and more strong computing power, and can also be a special gateway device with a wireless communication interface. The coordinator node mainly includes processor module, memory module, RF module, communication interface and keyboard module.

Among these modules, processor module mainly completes the analysis, fusion and preprocessing of the received data. Memory module is mainly used to save the relevant aircraft health data. RF module is the same as RF module in the terminal sensor node. The keyboard module is used to optimize the configuration parameters of the coordinator node. All kinds of communication interface mainly provide interface for data communication, complete the information exchange between the coordinator node and remote information center. Different from the common underlying terminal node, the coordinator node is rich in communication interface, which can be used to connect the wireless communication module, such as GPRS module, GPS and Beidou communication system. At the same time, it can also be directly connected through the RS232 serial port and PC host computer, and can be achieved on the coordination of internal aircraft health data and working state of the reading and writing through the PC. The coordinator node can also through RJ45 Ethernet access network, communicate through the public network and information center. Because the wireless communication is easy to be affected by environment and weather, there is a certain degree of instability, so it can be used as an alternate communication interface. In addition, there are a lot of the aircraft health data saved in the memory of coordinator node. It can use a common USB2.0 interface, data replication for permanent preservation, easy to analyze and query in the future.

\section{Protocol software design:- \\ Overall transport protocol architecture:-}

ZigBee wireless transmission technology based on IEEE802.15.4 communication protocol is used in wireless sensor networks for aircraft health monitoring. ZigBee wireless transmission technology work in band without registration, opening $2.4 \mathrm{GHz}$ industrial, scientific and medical. Its transmission rate is up to $250 \mathrm{kbit} / \mathrm{s} \sim 10 \mathrm{Mbit} / \mathrm{s}$, and transmission distance is up to $10 \sim 75 \mathrm{~m}$. If antenna gain is used, it's transmission distance can be increased to $1500 \mathrm{~m}$.

Compared with other wireless communication standards, the ZigBee protocol architecture constructed basing on open system interconnection (OSI) is compact. It includes physical layer (PHY) and media access control (MAC)defined in the IEEE 802.15.4 standard, as well as network layer (NWK) and application layer (APL) defined by the ZigBee alliance. APL framework includes application support sublayer (APS), ZigBee device object (ZDO) and the application object formulated by the manufacturer.

\section{Physical layer design:-}

The ZigBee physical layer defines the interface between the wireless transmission channel and the MAC layer, and provides the physical layer data service and the physical layer management service. Among them, the physical layer data service is to send and receive data from the wireless channel, while the physical layer management service maintains a database composed of physical layer related data. The function of physical layer data services includes the following five aspects: activation and dormancy for RF transceiver and channel energy detection, link quality indicator (LQI) of detection receive packets, clear channel assessment (CCA), and send and receive data. 
Channel energy detection mainly measures the power intensity of the received signal in the target channel, so as to provide the basis for channel selection for the network layer. LQI provides wireless signal strength and quality information when data frames are received for network layer or application layer. Different form channel energy detection, it is necessary to signal decode to generate a signal to noise ratios (SNR), and SNR is submitted to the upper layer with physical layer data units. CCA will determine if the channel is clear. IEEE 802.15.4 defines three CCA model: the first, simple judgment channel signal energy, when signal energy is lower than a threshold value that is clear channel. The second is by determining the characteristics of the wireless signal. This feature mainly includes two aspects, namely spread spectrum signal characteristics and carrier frequency. The third is the comprehensive of the first and the second mode, judging the channel weather clear or not based on the detection signal strength and signal characteristics.

Physical layer defines 3 carrier frequency bands using for sending and receiving data. There are some differences in the rate of data transmission, signal processing and modulation in the 3 frequency bands. The three frequency bands are $865 \mathrm{MHz}$ band 1 channel, $915 \mathrm{MHz}$ band 10 channels, $2.4 \mathrm{GHz}$ band 16 channels.

\section{MAC layer design:-}

IEEE 802.15.4 MAC layer includes setup, maintenance and disconnect of the radio link between devices, transmits and receives the frame, channel access and control, frame check and rapid automatic retransmission quest (ARQ), reserved time slot management and broadcast information management, etc. The main function of MAC layer when dealing with all of the physical layer wireless channel access is : the network coordinator to generate web beacons, synchronization with the beacon, support a area network (Pan) link establishment and disconnection, the equipment support for the safety, channel access method using CSMA access/collision avoidance (CSMA/CA) mechanism, processing and maintenance of guaranteed time slots (GTS) mechanism, provide a reliable communication link between two equal MAC entities, and so on.

The MAC layer provides an interface between the Service Specific Convergence Sub-layer (SSCS) and the physical layer [20]. MAC layer includes a management entity that can invoke the MAC layer management function through a service interface. In addition, the entity is also responsible for maintaining the management object database inherent in the MAC layer. At the MAC layer, two different service access point provide two different MAC layer service. That is, MAC layer provides data services by the common part sub-layer service access point. MAC layer provides management services through its management service access point.

IEEE 802.15.4 MAC layer defines the two basic channel access methods, respectively using for two different ZigBee network topology, which are the star network based on the control center and net network based on peer to peer control. In star network, center devices perform network formation and maintenance, time slot division, channel access control and dedicated bandwidth allocation function, and the rest of the equipment decide how to access and use the wireless channel according to broadcast information of the center equipment. It is a time slot carrier sense multiple access / collision avoidance (CSMA / CA) channel access algorithm. Peer-to-peer net network has no control of center equipment, also does not have the radio channel and broadcast information, is a standard CSMA / CA algorithm.

\section{Network layer design:-}

The main function of ZigBee network layer is to provide the necessary functions to ensure the normal work of MAC layer, and provide a suitable service interface for application layer [21]. In order to provide the interface to the application layer, network layer provides two functions service entities, which are data entity and management services entity.

The data entity in network layer provides services for data, transmitting data between two or more devices according to the format of application protocol data unit (APDU), and these devices must be in the same internal domain network. The data entity in network layer provides the following services:

To generate network layer protocol data unit (NPDU). The network layer data entity generates a protocol data unit in the network layer from the application support layer protocol data unit by adding an appropriate protocol header. 
To specify topology transport route. Network layer data entity can send a protocol data unit of network layer to a suitable device. The device may be the ultimate goal of communication equipment, and may also be a midst communication device in the communication link.

To secure. To ensure the authenticity and confidentiality of the communication.

The network layer management entity provides network management services that allow applications to interact with the stack.

The network layer management entity shall provide the following services:-

Configuring a new device. In order to ensure that the equipment is working properly, the equipment should have enough stack to meet needs of the configuration. Configuration options include a ZigBee coordinator or an initialization operation that is connected to a network device.

To initialize a network with the ability to build a new network.

To connect and disconnect the network. It is with the ability to connect or disconnect a network, as well as for the establishment of a ZigBee coordinator or router, and the ability to disconnect with network equipment requirements.

Addressing. ZigBee coordinator and router have the ability to allocate addresses for newly added devices.

To find neighbor device. It has the ability to find, record and report information about a neighbor's device.

To find route. It has ability to find and record the network routing for efficient delivery information.

To receive and control. It has the ability to control the device's receiving state, that is, when the receiver receives and the length of time, to ensure synchronization or normal reception of the MAC layer, etc.

\section{Application layer design:-}

Network application layer includes user application process (UAP) and application sub layer (ASL) [22]. Specifically, the application layer defines the software object interact with aircraft health monitoring process, and define related communications services to support real-time communication between multiple objects distributed applications in the aircraft health monitoring environment at the same time.

UAP is consist of one or more user application objects (UAO). In one device, UAO can realize local interactive communication service. UAO can also be further realized of interaction between different devices through the application layer. In a word, through the user application process, it can realize distributed applications for the realtime monitoring of aircraft health. The application layer can provide transparent data transmission service for UAP and device management application process (DMAP).

The main functions of UAP of aircraft health monitoring WSN include the following five aspects:

To collect aircraft health data information through the sensor. After preprocessing of the information, UAP operates the data or other equipment data to output, to complete the measurement of aircraft health data.

To generate and issuing alarm function. UAP of aircraft health monitoring finds aircraft health data over booking limit in monitoring, or generate alarm information when the state of UAO occurs switching.

By UAP, it can be realized interoperability with other field bus technology.

ASL mainly provides data transmission services and related management services for aircraft health. Data transmission services is promising user layer provides transparent data communication service by end to end, and supporting the client/server communication, releaser/subscriber communication and Report/Sink communication mode. Management services have support for local and remote management of the device. Through the application 
layer management services, it can remotely modify / set data in the application layer management information database.

\section{Network topology structure design:-}

\section{Selection of network topology structure:-}

The traditional wireless sensor monitoring network supports the star and peer network topology, namely the star structure, the net network structure and the tree structure, as shown in Figure 3. The star network, all nodes are communication with the central coordination, nodes cannot communicate directly, the central node has large energy consumption. It is suitable for less network node number, simple network structure, short range communications network application. The net network structure is a kind of irregular and arbitrary connections between nodes. Network topology has the main advantage of the high system reliability, but the structure is complex, it must use the routing algorithm and flow control algorithm. The tree topology has evoluted from the bus topology, shaped like a tree, the top as the root, root with branches, each branch can be taped branch. The advantages of the tree topology are easy to extend and isolate fault, but it all depends on the root node seriously. According to the characteristics of aircraft health monitoring, the tree structure is undoubtedly the best choice.

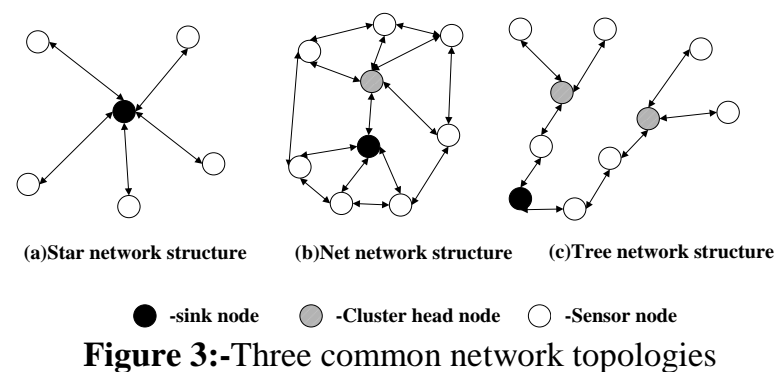

Isomorphism and heterogeneous selection:-

Wireless sensor network for aircraft health monitoring is a kind of network system with a wide range for real-time acquisition, transmission, analysis and processing of aircraft health parameters. For this network, due to the arrangement of many heterogeneous data of temperature, flow, pressure, strain, vibration basic sensor, and so on, after the pretreatment the data is sent to relevant users. Otherwise, the homogeneous network has maximum transmit power because each node transmits signal by omnidirectional. That is, all nodes are in receiving and sending node information, resulting in redundant paths exponentially increases during subsequent topology forming and eventually lead to rapidly shorten the network lifetime.

The network topology with heterogeneous structure has been divided nodes into different types and functions. It can reduce the number of unnecessary data transmission path, not only saving power, but also not appear too much repetition of the data transmission process, reduce implosion generation [23]. Heterogeneous structure can improve the movement function of the whole system, which results that the signal acquisition and information management system can adapt to more and more application requirements, address the needs of different types of signal acquisition, complete a specific task, has a higher adaptive ability and strong robustness. For aircraft health monitoring wireless sensor networks, heterogeneous network has the more power consumption than isomorphism network.

\section{Hybrid tree topology:-}

A hybrid tree network topology control structure is proposed in the paper, which is proposed by [24]. Firstly, the number of neighbor nodes is defined for each cluster head, and then the data transfer is carried out by hierarchical structure. At the same time, the network is composed of heterogeneous nodes. This design combines the characteristics of traditional star shape, net network architecture, and even tree topology, is a kind of multi-cluster heads and multi-layers wireless sensor network topology. The upper nodes in the system can be connected large-scale scattered isolated nodes in aircraft monitoring wireless sensor network area, and could also be implemented for classifying networking, so it is a certain flexibility degree, but also a larger space to expand on the number of nodes, 
and even can be extended to a hierarchical structure. This is consistent with the functional requirements, partition management and aircraft structure hierarchical characteristics of aircraft health monitoring.

The hybrid tree structure is divided into centralized network topology structure and distributed network topology structure. Both centralized and decentralized network topologies can increase the number of sink nodes appropriately according to the actual needs, so as to enhance the data processing ability of the sink node. In the centralized hybrid tree topology, and different from decentralized network topology, the cluster head node under one sink node can communicate with each other, but not communicate with other sink node's lower cluster head. Not only can exchange information between the sink nodes, but also if take over the information of other sink nodes which has enough ability.

The distributed network topology, data exchange does not occur between sink nodes, only the final data is sent to the gateway, and then the gateway makes the final treatment. Each cluster head node and adjacent cluster head in the network have information exchange, but all cluster heads do not form a complete closed loop structure, it can solve the cover problem of strip or tree nodes spread. At the same time, due to the wireless sensor network gateway in aircraft, the aircraft's flight will change the location, even in the process of change in the position, network signal and data transmission still can maintain the effective reception. The underlying sensor first collected information and data, then sent it to the cluster head node, cluster head node again preliminary data processing and forwarding with the adjacent cluster head nodes, and then sent it to sink node. Finally, the sink node completed comprehensive treatment.

To reduce the load of airborne computer, distributed network topology is more suitable for the demand for aircraft health monitoring. This structure has the advantages of simple connection, convenient maintenance, easy to expand, easy fault isolation. But the reliability is not high, any a workstation or link failure will affect the operation of the whole network, and the dependence to root of each node is too large. The communication path length is short, low cost, easy to expand node, more convenient to find a path. But in addition to the leaf nodes and its connected lines, the line fault of any node or connected to will make the system a great impact. A sink node has two working modes. One is response mode, namely passively trigged by the interest event or message sent by lower sensor nodes. The another is the active mode, that is, periodically scanning network and query the latest information of sensor nodes. The hybrid tree distributed network topology for aircraft health monitoring is shown in Figure 4.

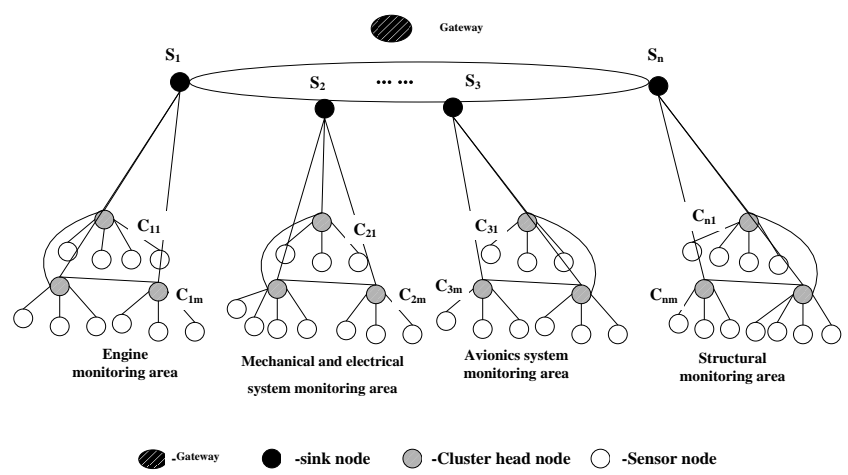

Figure 4:-The hybrid tree distributed network topology.

\section{Conclusion:-}

In view of wiring difficulties, poor flexibility, high cost, accident difficult to isolate and other practical problems for aircraft health state monitoring, combined with the characteristics of aircraft health monitoring function demand, regional management, heterogeneous data, using the advantages of wireless sensor network, a solutions based on wireless sensor network for aircraft health monitoring system is put forward, and the overall architecture, node hardware and protocol software are designed, a hybrid tree decentralized network topology structure is proposed for the aircraft health monitoring. How to carry out the network topology optimization control and energy management, data transmission, and enhance the reliability are the focus of the next step research. 


\section{Acknowledgment:-}

The author wish to thank those who had studied wireless sensor network,aircraft health monitoring, and structural health monitoring. This work was supported and inspired in part by a grant from them.

\section{Reference:-}

1. WANG Ping. Application Review of Blade Tip Timing Method for Foreign Aero-engine Blade Vibration Measurement[J]. Aeronautical Science \& Technology,2013,24(6):5-9.(in Chinese)

2. YANG Zhou, JING Bo, ZHANG Jie. Application of wireless Sensor Network on Airborne System of Prognostics and Health Management[J]. Journal of Computer Research and development.2011, 48 (Suppl.) : 338-342.

3. LIU Enpeng. Development and Application of Airborne Wireless Sensor Network Technology[J]. Test and control Technology, 2012, 31 (9) : 1-4.

4. Bai, H, Atiquzzaman, M, Lilja, D. Wireless Sensor Network for Aircraft Health Monitoring[C]. International Conference on Broadband Networks, DOI: 10.1109/BROADNETS.2004.92,2004.

5. Shang GAO, Xuewu DAI, Yu HANG, et al. Airborne Wireless Sensor Networks for Airplane Monitoring System[J]. Wireless Communications and Mobile Computing, https://doi.org/10.1155/2018/6025825,2018.

6. Wei Zhou, Bo Jing. A Study on the Wireless Sensor Networks MAC Protocol for Aircraft Health Monitoring. Lecture Notes in Electrical Engineering, 295:131-143 ,DOI: 10.1007/978-3-642-54174-2_12,2014.

7. Nikos Fragoulis, Vassilis Tsagaris, Vassilis Anastassopoulos. Information Fusion in Ad hoc Wireless Sensor Networks for Aircraft Health Monitoring. Engineering Against Fracture[M],DOI: 10.1007/978-1-4020-94026_25,2009.

8. Yu Lu, Al Savvaris, Antonios Tsourdos. Vibration energy harvesters for wireless sensor networks for aircraft health monitoring[C]. Metrology for Aerospace, DOI: 10.1109/MetroAeroSpace.2016.7573180,2016.

9. Zheng Jun Chew, Tingwen Ruan, Meiling Zhu. Strain Energy Harvesting Powered Wireless Sensor Node for Aircraft Structural Health Monitoring[C]. 30th Eurosensors Conference, EUROSENSORS 2016, pp.1717 - 1720.

10. Arms, S.W. Galbreath, J.H. Townsend,et al. Energy Harvesting Wireless Sensors and Networked Timing Synchronization for Aircraft Structural Health Monitoring[C]. International Conference on Wireless Communication, DOI: 10.1109/WIRELESSVITAE.2009.5172414,2009.

11. Rama K. Yedavalli, Rohit K. Belapurkar. Application of wireless sensor networks to aircraft control and health management systems[J]. Journal of Control Theory \& Applications, DOI: 10.1007/s11768-011-0242-9,2011.

12.Patterson, Tori Romell. Wireless Sensor Networks and Data Fusion for Structural Health Monitoring of Aircraft[J]. Dissertations \& Theses - Gradworks,2013.

13.Peng Yao, Gang Liu, Yan Liu. Critical Event-Triggered Transmission for Wireless Sensor Node in Aircraft Structural Health Monitoring[J]. Applied Mechanics and Materials, DOI: 10.4028/www.scientific.net/AMM.599601.856,2014.

14. Thomas Becker, Martin Kluge, Josef Schalk, et al. Autonomous Sensor Nodes for Aircraft Structural Health Monitoring[J]. IEEE Sensors Journal, Volume 9, Issue 11, pp: 1589 - 1595, Nov. 2009.

15.Losada, Markos \& Irizar, Andoni \& del Campo, Pablo \& Ruiz, Pedro \& Leventis, Apostolos. (2015). Design principles and challenges for an autonomous WSN for structural health monitoring in aircrafts. Proceedings of the 2014 29th Conference on Design of Circuits and Integrated Systems, DCIS 2014. 10.1109/DCIS.2014.7035595.

16.Luís M. L. Oliveira, Joel J. P. C. Rodrigues, Bruno M. Mação, et al. A WSN Solution for Light Aircraft Pilot Health Monitoring[C]. Wireless Communications \& Networking Conference 2012, DOI: $10.1109 /$ WCNC.2012.6213959,2012.

17.ZHANG Chuanchao, CHEN Li. Research on Key Technologies of Wireless Sensor Network for Aircraft Health Monitoring[J]. Applied Mechanics and Materials, Trans Tech Publications,2014.

18. Ma Ning, Lu Chen. Research on PHM architecture of aircraft[J]. J. Huazhong Univ. o f Sci. \& Tech. (Natural Science Edition). Vo 1. 37 Sup. Aug. 2009: 207-209.

19. AVIC Shanghai Aero Measurement-controlling Research institute. Aviation Fault Diagnosis and Health Management Technology[M]. Beijing: Aviation Industry Press,2013. 
20. Koubaa A, Alves M, Nefzi B, Song Y Q. Improving the IEEE802.15.4 slotted CSMA/CA MAC for time-critical events in wireless sensor networks. The 5th International Workshop on Real-Time Networks (RTN'06) , Dresden, Germany, July ,5-7, 2006.

21.Gutierrez J A, Callamay E, Mitter V, et al. IEEE802.15.4 - A development standard for low power, low-cost wireless personal area networks. IEEE Network Magazine,2001,15(5):12-19.

22. The Zigbee Specification version 1.0 (Q4/2007), Zigbee Alliance.

23. Gerla M, Kleinrock L. On the topological design of distributed computer networks. IEEE trans. on Commun., 1997,25(1):48-60.

24.ZHOU Ying. Research on Topology Control for Wireless Sensor Networks[D]. Wuhan: Wuhan University of Technology,2007. 